\title{
Reentrant dynamics of driven pancake vortices in layered superconductors
}

\author{
H. J. Zhao, ${ }^{1,2, *}$ Wenjuan Wu, ${ }^{1,3}$ Wei Zhou, ${ }^{1}$ Z. X. Shi, ${ }^{1, \dagger}$ V. R. Misko, ${ }^{2}$ and F. M. Peeters ${ }^{2}$ \\ ${ }^{1}$ Department of Physics, Key Laboratory of MEMS of the Ministry of Education, Southeast University, Nanjing 211189, China \\ ${ }^{2}$ Department of Physics, University of Antwerpen, Groenenborgerlaan 171, B-2020 Antwerpen, Belgium \\ ${ }^{3}$ College of Science, Nanjing Forestry University, Nanjing 210037, China
}

(Received 11 March 2016; revised manuscript received 31 May 2016; published 18 July 2016)

\begin{abstract}
The dynamics of driven pancake vortices in layered superconductors is studied using molecular-dynamics simulations. We found that, with increasing driving force, for strong interlayer coupling, the preexisted vortex lines either directly depin or first transform to two-dimensional (2D) pinned states before they are depinned, depending on the pinning strength. In a narrow region of pinning strengths, we found an interesting repinning process, which results in a negative differential resistance. For weak interlayer coupling, individually pinned pancake vortices first form disordered 2D flow and then transform to ordered three-dimensional (3D) flow with increasing driving force. However, for extremely strong pinning, the random pinning-induced thermal-like Langevin forces melt 3D vortex lines, which results in a persistent 2D flow in the fast-sliding regime. In the intermediate regime, the peak effect is found: With increasing driving force, the moving pancake vortices first crystallize to moving 3D vortex lines, and then these 3D vortex lines are melted, leading to the appearance of a reentrant $2 \mathrm{D}$ flow state. Our results are summarized in a dynamical phase diagram.
\end{abstract}

DOI: 10.1103/PhysRevB.94.024514

\section{INTRODUCTION}

The dynamics of a system of particles in the presence of pinning have attracted considerable attention over the past decades. Such systems include: Wigner crystals [1-3], interacting colloids, charged-density waves [4], skyrmions, crystal defects, etc. One of the most studied systems within this class is vortices in type-II superconductors. Various dynamical phases including pinned states, elastic flow, plastic flow, and fast-sliding flow were revealed in experiments [5-9] and in theory [10-15]. In general, these rich phases are determined by the interplay among the driving, pinning, thermal forces, and the elastic force due to the vortex-vortex interaction. In systems with regular pinning arrays, $N$-shaped [16-19] and $S$-shaped $[18,19]$ negative differential resistance (NDR) has been revealed. In low- $\kappa$ or multiband superconductors, such as $\mathrm{MgB}_{2}$, the intervortex interaction is determined by competing attractive and repulsive forces instead of a pure repulsive force as is usual in type-II superconductors [20-25]. Driving of such media with competing interaction reveals a rich variety of dynamical regimes including disordered motion, moving clusters, and moving ordered stripes [26]. The formation of stripes is strongly related to the strength of the pinning: Longitudinal (i.e., formed in the direction of the driving force) or transverse (i.e., formed in the direction normal to the driving) stripes can be formed in the weak or strong pinning regime, respectively [26].

In multilayered superconductors [27] and layered anisotropic superconductors, including superconducting cuprates and recently discovered high- $T_{c}$ iron-based superconductors, vortex lines are weakly coupled in the direction perpendicular to the superconducting layers. As a result, random pinning or thermal fluctuation may be able to plastically deform the straight vortex lines or even tear these vortex lines

\footnotetext{
*haijunzhao@seu.edu.cn

${ }^{\dagger}$ zxshi@seu.edu.cn
}

and lead to the formation of pancake vortices as a result of the decoupling transition of vortex lines [28-31]. A similar transition may also occur in type-II superconductors when the applied magnetic field is close to the upper critical field $H_{c 2}$ where vortex lines become soft and can be easily bent $[32,33]$. These bent vortex lines, or pancake vortices, can much better adjust themselves to the random pinning positions, thus leading to a large increase in the critical current [30] or to the appearance of reentrant pinning phases [32,33]. A similar behavior can also be observed in multiband superconductors where a single vortex is composed of contributions from each superconducting condensate [22,34]. By applying an external driving force, the different components can be separated as a result of the dissociation transition, similar to the decoupling transition in layered superconductors [35].

However, to study the above-mentioned decoupling transition or the dissociation transition numerically, one has to employ a three-dimensional (3D) model or multicomponent two-dimensional (2D) models, which makes the problem much more complicated as compared to the 2D case. Moreover, the underlined physics is different for $2 \mathrm{D}$ and $3 \mathrm{D}$ cases. For the 2D case, it is determined by two factors: the intervortex interaction which forces the vortices to form a triangular lattice (or clusters and stripes in low- $\kappa$ or multiband superconductors) and the vortex-pinning force which locally deforms the triangular lattice adjusting it to the pinning sites that results in energetically favorable vortex structures. However, for the 3D case, the intervortex interaction includes repulsion between vortices within the same layer and an attraction between vortices within different layers. The former favors the arrangement of vortices in a triangular lattice, whereas the latter tends to line up the vortices. In the 3D case, the vortex-pinning force not only disorders in-layer orderings, i.e., triangular lattices, but also interlayer ordering, i.e., vortex lines. Olson and co-workers numerically studied vortex dynamics phases of vortices in layered superconductors with strong uncorrelated pinnings $[30,36]$. A number of dynamical regimes including 2D(3D) pinning, 2D(3D) plastic flow, 3D smectic, and 3D reordered 
flow were revealed by varying the interlayer coupling strength and external driving force. However the pinning force strength was constant in their studies. It is worth noting that the coexistence of both the repulsive and the attractive interactions makes this system similar to a $2 \mathrm{D}$ system of driven particles with repulsive-attractive competing interactions where the static and dynamical properties strongly depend on the interparticle interaction parameters as well as the pinning strength [24,26]. Therefore, a system of vortices in layered superconductors (or another similar system of interacting particles) is expected to display new phenomena for weak or intermediate pinning regimes, which needs further investigation.

In this paper, we employ a 3D molecular-dynamics (MD) simulation to study the vortex dynamics in a layered superconductor. We reveal various dynamical regimes which strongly depend on the interlayer coupling strength $s_{m}$, pinning strength $f_{p}$, and the applied driving force $f_{d}$. Our results show several interesting phenomena including a NDR caused by a repinning process and a reentrant disordered 2D flow phase for strong $f_{p}$ and small $s_{m}$. Based on our results, a dynamical phase diagram is constructed on the plane " $s_{m}$ versus $f_{p}$." The paper is organized as follows. In Sec. II, we describe the model. Various dynamical regimes are studied in Sec. III. In Sec. IV, we present the phase diagram showing the revealed dynamical phases. The conclusions are presented in Sec. V.

\section{MODEL}

We study the dynamics of pancake vortices in layered superconductors when an external magnetic field is applied perpendicular to the superconducting plane by numerically integrating the Langevin equation of motion using moleculardynamics simulations. We employ periodic boundary conditions in the $x$ and $y$ directions and open boundary conditions in the $z$ direction. The motion of pancake vortices is described by the following overdamped equation:

$$
\eta \mathbf{v}_{i}=-\sum_{j=1}^{N_{v}} \nabla U\left(\rho_{i, j}, z_{i, j}\right)+\mathbf{F}_{i}^{p}+\mathbf{F}_{i}^{T}+\mathbf{F}_{d}
$$

Here $\eta$ is the viscosity coefficient, $\mathbf{v}_{i}$ is the velocity of the pancake vortex labeled $i, N_{v}$ is the total number of pancake vortices, and $\rho_{i, j}, z_{i, j}$ is the distance between vortex $i$ and vortex $j$ in cylindrical coordinates. The intervortex interaction energy within the same layer is [30,36-38]

$$
U\left(\rho_{i, j}, 0\right)=2 d \epsilon_{0}\left[\left(1-\frac{d}{2 \lambda}\right) \ln \frac{R}{\rho_{i, j}}+\frac{d}{2 \lambda} E_{1}\right],
$$

and between layers,

$$
U\left(\rho_{i, j}, z\right)=-s_{m} \frac{d^{2} \epsilon_{0}}{\lambda}\left[\exp \left(-\frac{z}{\lambda}\right) \ln \frac{R}{\rho_{i, j}}+E_{2}\right],
$$

where $\epsilon_{0}=\Phi_{0}^{2} /(4 \pi \lambda)^{2}, \Phi_{0}=h c / 2 e$ is the elementary flux quantum, $d$ is the interlayer spacing, which equals to thickness of pancake vortices, $\lambda$ is the London penetration depth, $R$ is the maximum radial distance, $E_{1}=\int_{\rho}^{\infty} d \rho^{\prime} \exp \left(-\rho^{\prime} / \lambda\right) / \rho^{\prime}$, and $E_{2}=\int_{\rho}^{\infty} d \rho^{\prime} \exp \left(-\sqrt{z^{2}+\rho^{\prime 2}} / \lambda\right) / \rho^{\prime}$. Here, $s_{m}$ is the interlayer coupling strength, and we have neglected vortex interactions due to Josephson coupling, which is valid for highly anisotropic superconductors, such as bismuth strontium calcium copper oxide [37]. The long-range logarithm interaction is treated using methods of Ref. [39]. $\mathbf{F}_{i}^{p}$ is the interaction of the $i$ th vortex with the pinning sites $[30,36]$,

$$
\mathbf{F}_{i}^{p}=-\frac{2 d \epsilon_{0}}{\lambda} f_{p} \sum_{j=1}^{N_{p}}\left(\frac{\mathbf{r}_{i}-\mathbf{r}_{k}^{(p)}}{r_{p}}\right) \Theta\left(\frac{r_{p}-\left|\mathbf{r}_{i}-\mathbf{r}_{k}^{(p)}\right|}{\lambda}\right) .
$$

Here, $N_{p}$ is the number of pinning sites in the layer where vortex $i$ is located, $\mathbf{r}_{k}^{(p)}$ is the location of the $k$ th pinning site in this layer, and $\Theta$ is the Heaviside step function. $f_{p}$ and $r_{p}$ are the pinning strength and pinning radius, respectively. In our simulations, we set pinning sites to be located at uncorrelated random positions, and their densities in different layers are the same.

The thermal stochastic term $F_{i}^{T}$ obeys the following conditions:

$$
\left\langle F_{i}^{T}(t)=0\right\rangle
$$

and

$$
\left\langle F_{i}^{T}(t) F_{i}^{T}\left(t^{\prime}\right)\right\rangle=2 \eta k_{B} T \delta_{i, j} \delta\left(t-t^{\prime}\right) .
$$

Here, $\mathbf{F}_{d}=\left(2 d \epsilon_{0} / \lambda\right) f_{d} \hat{\mathbf{e}}_{x}$ is the external driving force generated by the applied supercurrent, and $\hat{\mathbf{e}}_{x}$ is a unit vector in the $x$ direction. In order to characterize the interlayer coupling of pancake vortices, we calculate the correlation function in the $z$ direction, which is defined as [30]

$$
\begin{aligned}
c_{z}= & 1-\left\langle\left[\left|\mathbf{r}_{i, L}-\mathbf{r}_{i, L+1}\right| /\left(a_{0} / 2\right)\right]\right. \\
& \left.\times \Theta\left[\left(a_{0} / 2-\left|\mathbf{r}_{i, L}-\mathbf{r}_{i, L+1}\right|\right) / \lambda\right]\right\rangle .
\end{aligned}
$$

Here $a_{0}$ is the in-plane vortex lattice constant, $\left|\mathbf{r}_{i, L}-\mathbf{r}_{i, L+1}\right|$ is the distance between two vortices in two neighboring layers. For the in-layer ordering, we calculate the radial distribution function (RDF),

$$
g(r)=\frac{1}{N} \sum_{i=1}^{N} \frac{\Delta N / \Delta r}{2 \pi r} .
$$

Here, the lower index indicates that the RDF centers at the position of the $i$ th particle, $\Delta N$ is the number of particles whose distance to the $i$ th particles is between $r$ and $r+\Delta r$, and $N$ is the total number of vortices in the system. The RDF describes the variation of the particle density as a function of the distance. Below we present our results for various pinning forces $f_{p}$ and interlayer coupling strengths $s_{m}$. For every new run of simulations, we generate new locations of random pinning centers. We fixed the following interaction parameters: the size of simulation box $l_{x}=l_{y}=16$, pancake vortex density $n_{v}$, the interlayer distance $d=0.005$, radius of pinning sites $r_{p}=0.2$, density of pinning $n_{p}=2.08$, and number of layers $N_{\text {layer }}=8$ (we also used other parameters and found that either the system is insensitive to their change, or similar effects can be achieved by changing the above parameters).

\section{VORTEX DYNAMICS}

To study the vortex dynamics, we start from a stable configuration and then turn on the driving force $f_{d}$. We set the temperature to zero (the effect of finite temperatures will be analyzed elsewhere). The driving force $f_{d}$ is gradually 
increased from zero with steps of $\delta f_{d}=0.001$. For each value of the driving force, we wait typically $5 \times 10^{5}$ simulation steps to make sure the system is relaxed to the steady state. The dynamics of the system is determined by four main factors: the in-layer repulsive and interlayer attractive vortex-vortex interactions, vortex-pinning interactions, and the applied driving. Here, we fix the pancake vortex density $n_{v}=$ 0.313 , i.e., the in-layer repulsive vortex-vortex interaction is almost unchanged. To characterize the dynamical regimes, we calculate the average velocity $\langle v\rangle$ and correlation functions $c_{z}$ as a function of $f_{d}$ for varying $s_{m}$ and $f_{p}$. In general, vortices are all pinned when $f_{d}$ is small and $\langle v\rangle=0$. In the opposite case, they are all depinned when $f_{d}$ is large $\left(>f_{p}\right)$ and $\langle v\rangle \propto f_{d}$. For very small $f_{d}$, the pinned state can either be composed of coupled vortex lines (3D) or be composed of decoupled pancake vortices (2D), depending on the strength of interlayer coupling strength $s_{m}$. In the opposite case of very large $f_{d}$, pancake vortices are normally reordered in $3 \mathrm{D}$ vortex lines. The driving force may either tear preexisted coupled vortex lines (decoupling transition), which leads to a decrease in $c_{z}$ or help depinned pancake vortices couple to each other and form vortex lines (coupling transition), which leads to an increase in $c_{z}$. The depinning (repinning) transitions and decoupling (coupling) transitions correspond to dramatic changes in the curves of $\langle v\rangle$ and $c_{z}$, respectively. These transitions indicate different dynamical regimes. Below, we present our results in two subsections. In the first subsection, the interlayer coupling strength $s_{m}$ is strong, i.e., sufficient to support 3D vortex lines at zero driving. The opposite case of a weak $s_{m}$ when 2D initial states are formed is discussed in the second subsection. In each subsection, we consider different pinning regimes.

\section{A. Strong interlayer coupling: depinning of preexisted vortex lines}

In Fig. 1(a), we show typical curves of $\langle v\rangle\left(f_{d}\right)$ and $c_{z}\left(f_{d}\right)$ for weak pinning $\left(f_{p} \leq 0.4\right.$ for $s_{m}=32$ ) when its force is comparable to the elastic force due to in-layer repulsive vortexvortex interactions. With increasing $f_{d}, c_{z}$ first increases and then becomes saturated at the depinning point. Therefore, there are only two dynamical regimes: 3D pinned and 3D flow. The increase in $c_{z}$ before the depinning indicates the healing process, i.e., the driving force first heals pinning-induced elastically deformed vortex lines. Note that, similar healing processes were also found in systems with both regular and random pinning centers [40]. With decreasing $f_{d}$, no obvious hysteretic effect is observed for $\langle v\rangle . c_{z}=1$ for all driving, i.e., these highly ordered vortex lines still exist after the depinning-pinning transition.

For intermediate pinning $\left(0.4 \leq f_{p} \leq 1\right.$ for $\left.s_{m}=32\right)$, the pinning forces are much larger than typical elastic forces due to in-layer repulsive interaction, i.e., they are sufficient to largely deform the triangular lattices. As shown in Fig. 1(b), there are two transition points on $c_{z}$ but only one depinning point on $\langle v\rangle$. The transition points on $c_{z}$ include a $3 \mathrm{D}$ to $2 \mathrm{D}$ decoupling transition point that appears in the pinned regime and a $2 \mathrm{D}$ to $3 \mathrm{D}$ coupling transition point that appears in the depinned regime (note that both transitions are very sharp). As a result, four vortex dynamical regimes including 3D pinned, 2D pinned, 2D flow, and 3D flow are involved. These dynamical regimes agree with the ones reported in Ref. [30]. With decreasing $f_{d}$, similar to the weak pinning case, no hysteretic effect appears in $\langle v\rangle$. However, a strong hysteretic effect is seen in $c_{z}$ : Decreasing $f_{d}$ results in a supercooled 2D pinned state when $f_{d}$ is close to zero instead of 3D pinned states.

Besides the above two cases, there is also a crossover region with quite different dynamics when $f_{p}$ and $s_{m}$ are limited within a very narrow area. In Figs. 2(a)-2(c), we plot the average velocity $\langle v\rangle$, the fraction of depinned vortices $\gamma$, and the correlation function $c_{z}$ as a function of $f_{d}$ for $f_{p}=0.38$ and $s_{m}=18$. As one can see, there are two depinning points and only one repinning point on $\langle v\rangle$. One of the depinning points is located in the $3 \mathrm{D}$ regime. Another one is located at the $2 \mathrm{D}$ to $3 \mathrm{D}$ transition point, i.e., at the same position of the $2 \mathrm{D}$ to $3 \mathrm{D}$ coupling transition. Similarly, the repinning
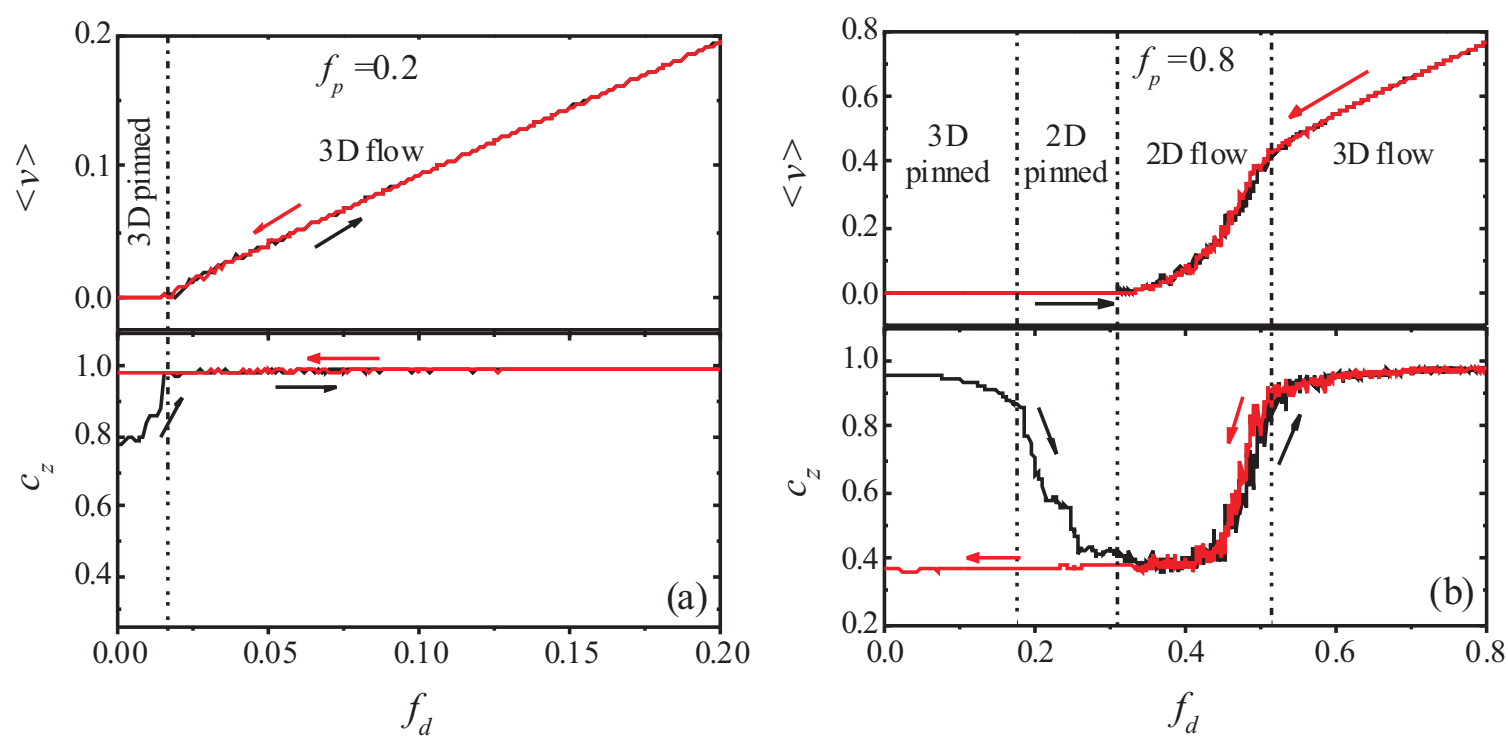

FIG. 1. The average velocity $\langle v\rangle$ (upper panels) and correlation function $c_{z}$ (lower panels) versus driving force $f_{d}$ for coupling strength $s_{m}=32$, and pinning strength (a) $f_{p}=0.2$ and (b) $f_{p}=0.8$. Black (red) lines correspond to increasing (decreasing) $f_{d}$. 


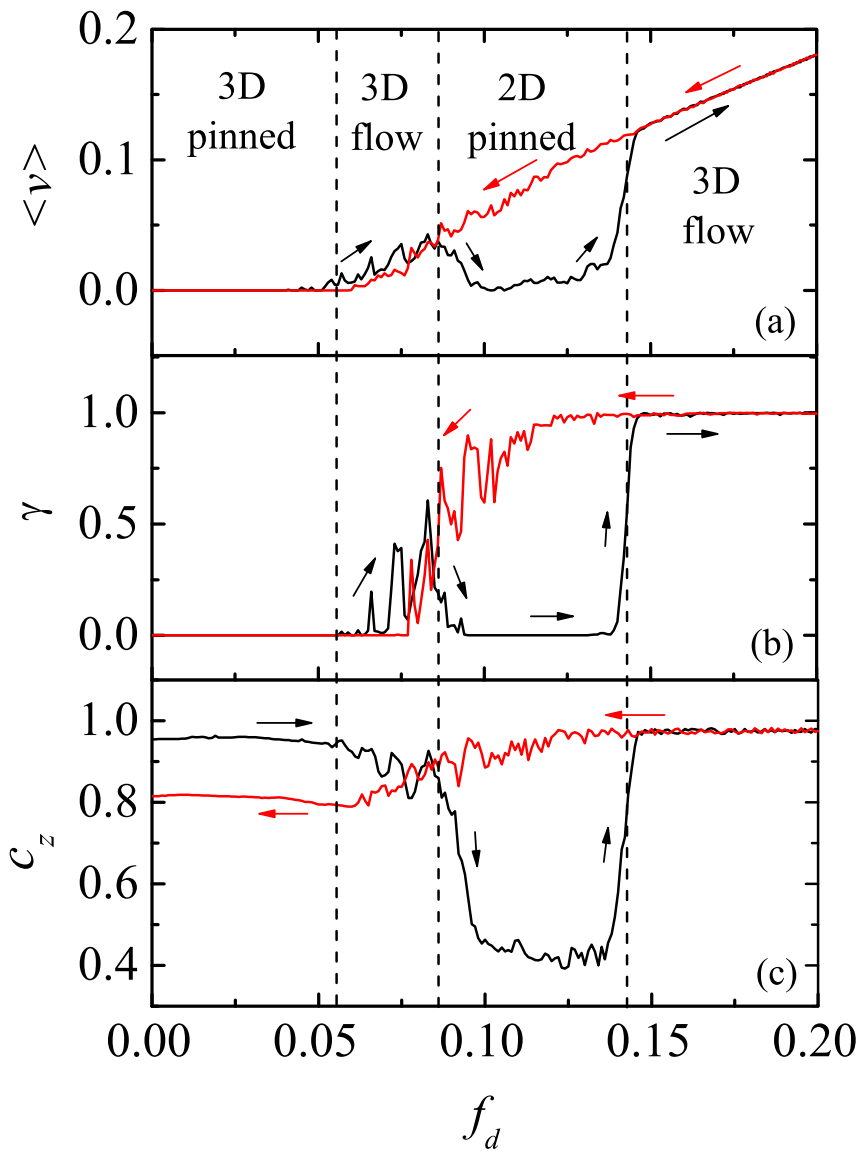

FIG. 2. The average velocity (a) $\langle v\rangle$, the fraction of depinned vortices, (b) $\gamma$, and the correlation function (c) $c_{z}$ versus driving force $f_{d}$ for $f_{p}=0.38$ and $s_{m}=18$. Black (red) lines correspond to increasing (decreasing) $f_{d}$.

point appears at the same position of $3 \mathrm{D}$ to $2 \mathrm{D}$ decoupling transition. Such a repinning process reveals an interesting NDR effect, which was also found in vortex systems with regular pinnings [16-19] and a reentrant pinning behavior, which was experimentally observed $[32,33]$. Such a phenomenon was also reported in layered superconductors when vortex lines decouple to pancake vortices [36]. However, the vortex line states found in Ref. [36] were artificially overcooled or overheated states instead of the real dynamical steady states in our case. These transition points separate four dynamical regimes: 3D pinned, 3D flow, 2D pinned, and 3D flow again. Note that here the 2D flow regime is very narrow or nearly missing. One can assume that the first appearing 3D flow may be caused by the occasional motion of a few individual vortex lines. As shown in Fig. 2(b), the fraction of depinned vortices $\gamma$ reaches a maximum value $(>0.5)$ in this regime, i.e., more than half of the vortices are depinned, indicating collective motion of most vortices. With decreasing $f_{d}$, a large $c_{z}$ with a little decrease at small $f_{d}$ indicates that vortex lines are always formed and $\langle v\rangle$ almost linearly decreases with $f_{d}$, which results in a strong hysteretic effect on both $\langle v\rangle$ and $c_{z}$.

The physics behind this unusual NDR phenomenon or reentrant pinning behavior is the following: When $f_{d}$ is increased from zero, first, the preexisting vortex lines are depinned. Further increasing $f_{d}$ induces the decoupling transition. After the tearing of the vortex lines, pancake vortices can adjust to random pinnings much better, which greatly enhances pinning, i.e., causes the repinning behavior. The repinning and decoupling transitions appear simultaneously since they are caused by the same physics. With further increasing $f_{d}$, the driving force becomes sufficient to depin 2D pinned vortices. Once this depinning process occurs, the depinned pancake vortices immediately couple to each other and form vortex lines, i.e., the $2 \mathrm{D}$ pinned state is directly transformed to the 3D flow state. The missing 2D flow regime indicates that the 2D pinned state is a dynamically induced supercooled state (which can also be proven by the strong hysteretic effect), and the most stable state at the coupling transition point is the 3D flow. The supercooled 2D pinned state suddenly relaxes to the 3D flow state when the transition is turned on by depinning of a single pancake vortex. The fast relaxation process results in a jump over the intermediate states, i.e., 2D flow state, which is therefore missing. When slightly decreasing $s_{m}$ (or increasing $f_{p}$ ), the coupling force becomes insufficient to support a 3D flow before it transforms to $2 \mathrm{D}$ pinned states. In the opposite case, slightly increasing $s_{m}$ (or decreasing $f_{p}$ ), $c_{z}$ can hardly decrease to 0.4 , which are typical values for completely decoupled 2D states. Thus, in this situation both individual pancake vortices and vortex line segments exist. These segments are much easier to be depinned than pancake vortices. As a result, the supercooled 2D pinned state becomes unstable, which also can lead to the disappearance of NDR. For the above reasons, NDR is only found in a very narrow region of $s_{m}$.

For very strong pinning, i.e., when the pinning forces are much larger than the in-layer vortex-vortex interaction force, the formation of 3D states requires a very large $s_{m}$, which will make the interlayer vortex-vortex attractive interaction stronger than the in-layer repulsive interaction. Therefore, the net force between two neighboring vortex lines becomes an attractive-repulsive competing force instead of pure repulsive forces. This competing force leads to the formation of clusters or stripes instead of triangular lattices in the absence of pinning $[24,25]$. Such conditions may exist in other physical systems but not vortices. Therefore, we do not consider this situation here.

\section{B. Weak interlayer coupling: depinning of individual pancake vortices}

In Fig. 3(a), we show the functions $\langle v\rangle\left(f_{d}\right)$ and $c_{z}\left(f_{d}\right)$ for the weak pinning case $\left(f_{p} \leq 0.8\right.$ for $\left.s_{m}=2\right)$. As one can see, each of these two curves has one transition point: a depinning point on curve $\langle v\rangle$ and a decoupling point on $c_{z}$. These two transition points are located on these curves at different $f_{d}$ 's, i.e., the transitions are uncorrelated. As a result, three different regions appear in $f_{d}$ which define three dynamical regimes including: 2D pinned, 2D flow, and 3D flow. For increasing values of $f_{p}$, i.e., in the intermediate pinning regime $\left(0.8<f_{p} \leq 2.2\right)$, similar dynamical regimes are found. However, in this case there are three main differences: First, the coupling transition point appears for $f_{d}>f_{p}$, i.e., in the fast-sliding regime where all the vortices are depinned; second, here $c_{z}$ increases much more slowly as compared to the weak pinning case, which results in very broad crossover regimes where both $2 \mathrm{D}$ and 

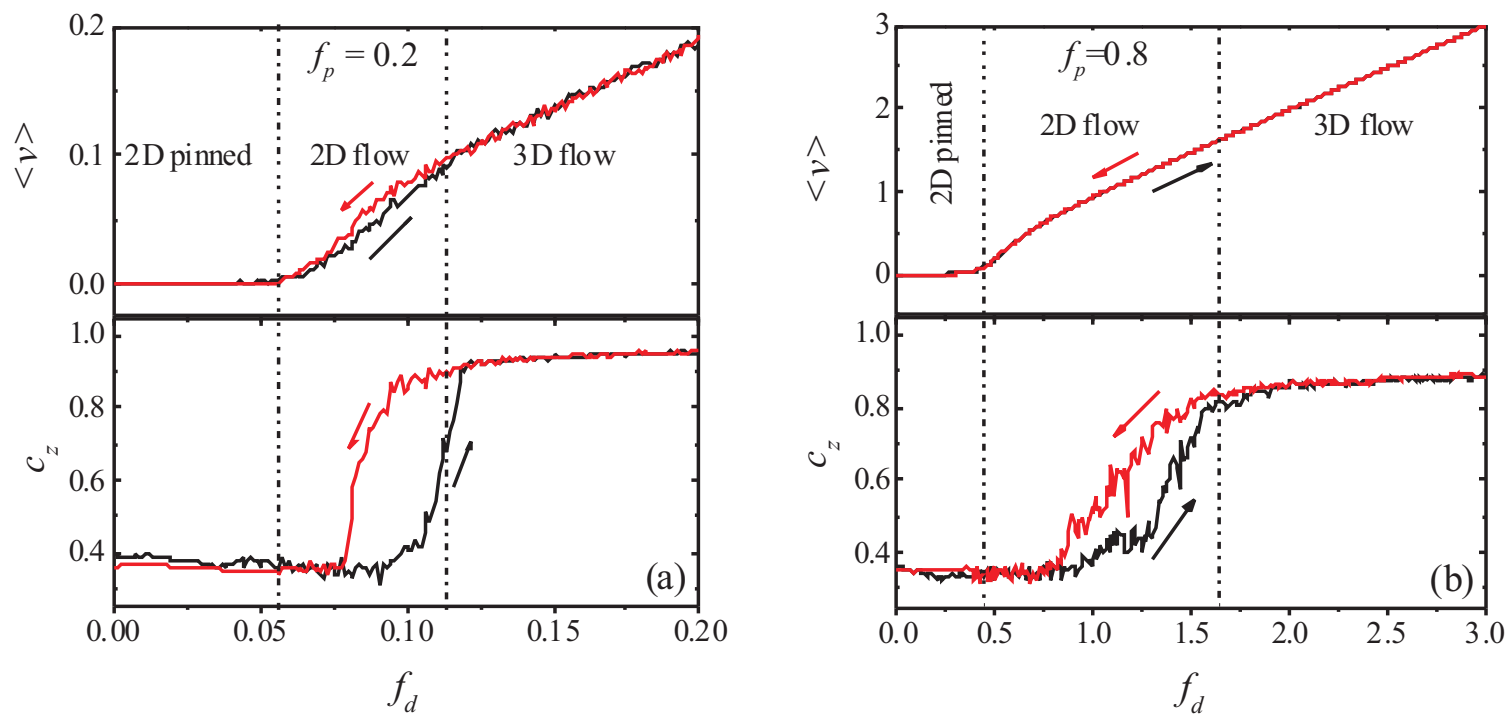

FIG. 3. The average velocity $\langle v\rangle$ (upper panels) and correlation function $c_{z}$ (lower panels) versus driving force $f_{d}$ for coupling strength $s_{m}=2$ and pinning strength (a) $f_{p}=0.2$ and (b) $f_{p}=0.8$. Black (red) lines corresponding to increasing (decreasing) $f_{d}$.

3D flows are involved; third, the saturated value of $c_{z}$ at large $f_{d}$, i.e., deep in the fast-sliding regime, is less than $1(\approx 0.8)$, indicating that vortex lines are still partially deformed.

For very strong pinning $\left(f_{p}>2.2\right.$ for $\left.s_{m}=4\right)$ as shown in Fig. 4(a) with increasing $f_{d}$, a well-pronounced peak appears on the curve of $c_{z}$. Namely, with increasing $f_{d}$, first, the system shows a jump from the $2 D$-flow dynamical regime to the ordered $3 D$ flow and then turns by gradually disordering back to the 2D flow. The position of this peak is located at $f_{d}=5.8>2 f_{p}$, i.e., deep in the fast-sliding regime. In Figs. 4(b)-4(e), we show 2D projections of all the pancake vortices within different layers. It is clear that for $f_{d} \approx f_{p}$, i.e., just after depinning, both the projections [see Fig. 4(b)], and the small $c_{z}$ values indicate that individual pancake vortices instead of vortex lines (i.e., 2D states) are formed. For $f_{d} \approx 5.8$, i.e., close to the peak, the overlapped dots in Fig. 4(c) show projections of coupled vortex lines, i.e., formation of a 3D state, which also agrees with the large peak value of $c_{z}$. Moreover, these dots are arranged into triangles, i.e., triangular lattices are formed. Note that one of the triangular lattices' orientation is in the longitudinal direction. For $f_{d} \approx 9.0$, i.e., in a region just after the peak, these overlapped dots turn to longitudinal stripes [see Fig. 4(d)], which means vortex lines are partially melted or stretched in the longitudinal direction. Finally, for very large $f_{d}$, the projections [see Fig. 4(e)] and $c_{z}$ are similar to these for $f_{d} \approx f_{p}$ [see Fig. 4(b)], i.e., a reentrant $2 \mathrm{D}$ flow state is revealed. Note that, unlike the case for intermediate pinning (where the appearance of NDR is
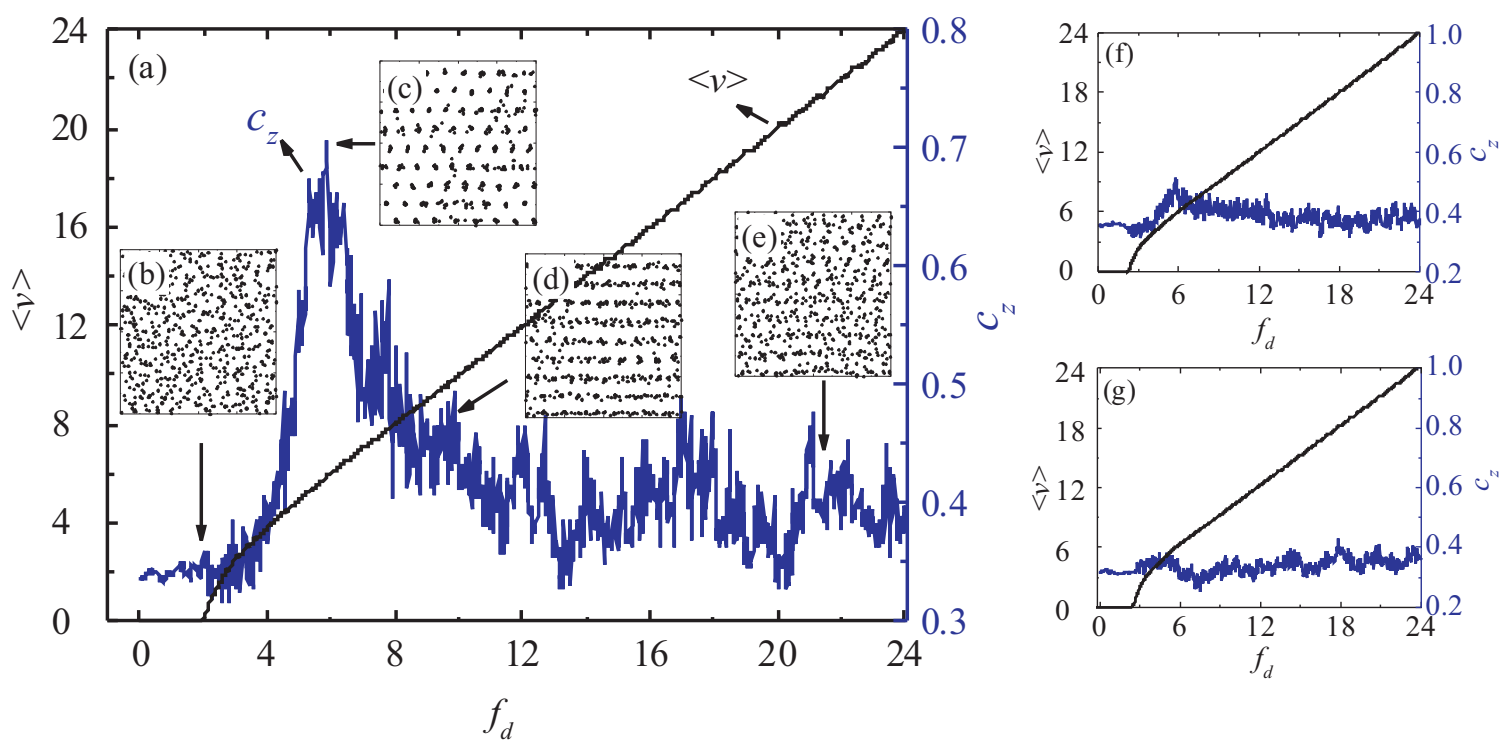

FIG. 4. The average velocity $\langle v\rangle$ (black line) and correlation function $c_{z}$ (blue/gray line) versus driving force $f_{d}$ for coupling strength $s_{m}=4$ and pinning strength (a) $f_{p}=2.7$, (f) $f_{p}=3.0$, and (g) $f_{p}=3.5$. Projections of the positions of pancake vortices for (b) $f_{d}=1.84$, (c) $f_{d}=5.84$, (d) $f_{d}=9.02$, and (e) $f_{d}=21.3$. 

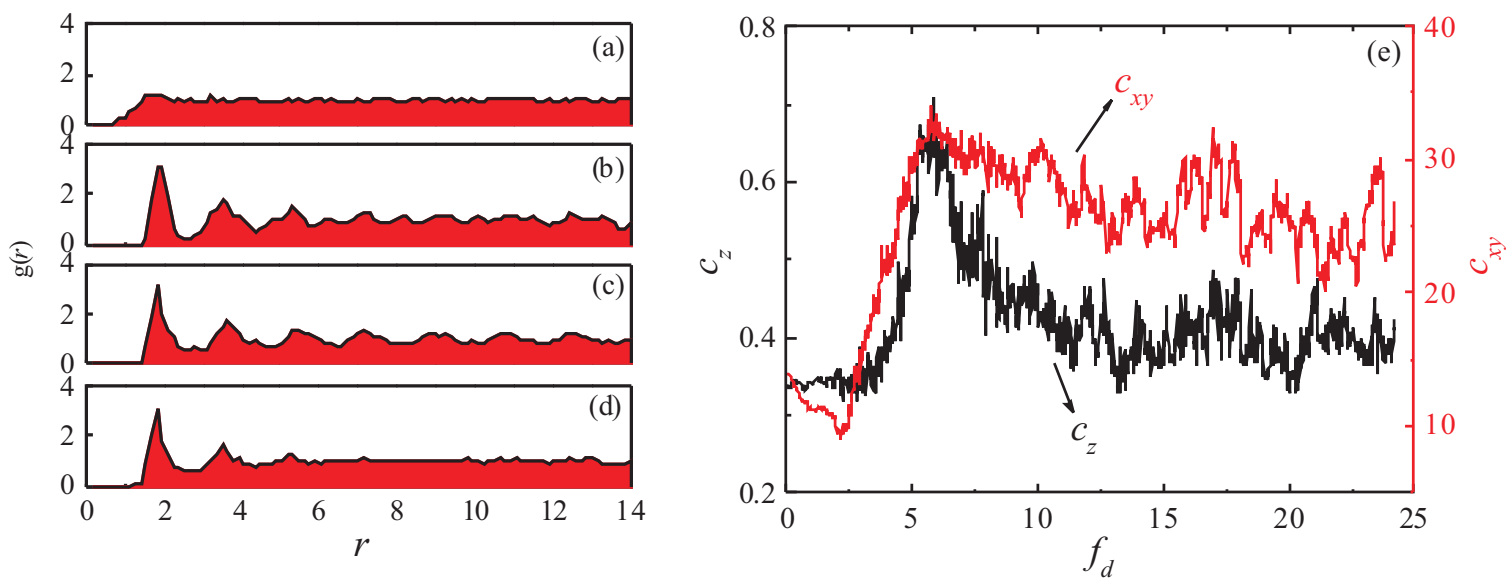

FIG. 5. The RDF for (a) $f_{d}=1.84$, (b) $f_{d}=5.84$, (c) $f_{d}=9.02$, and (d) $f_{d}=21.3$, corresponding to projections shown in Figs. 4(b)-4(e), respectively. (e) The correlation functions $c_{x y}$ (red/gray line) and $c_{z}$ (black line) versus driving force $f_{d}$ for coupling strength $s_{m}=4$ and pinning strength $f_{p}=2.7$.

detected) in which dramatic changes in $\langle v\rangle$ appear at the point of the coupling or decoupling transition, here, $\langle v\rangle$ is always proportional to $f_{d}$, i.e, such transition cannot be observed experimentally by measuring $I-V$ curves. For larger $f_{p}$, this peak becomes smaller [see Fig. 4(f)]. For even large $f_{p}$, it disappears, resulting in a persistent 2D flow [see Fig. 4(f)].

To characterize ordering within the $x y$ plane, we calculate the RDF within each layer and then calculate an averaged RDF. In Figs. 5(a)-5(d), we show the RDFs of the corresponding configurations shown in Figs. 4(b)-4(e). The first RDF [Fig. 5(a)] corresponding to Fig. 4(b) exhibits poor peaks, indicating that pancake vortices are randomly distributed within each layer. The second RDF [Fig. 5(b)] corresponding to Fig. 4(c) exhibits well-pronounced peaks. These peaks appear at long distances, which is indicative of pronounced long-range ordering. Therefore, 3D moving crystals (or Bragg glasses more precisely) are formed. Such long-range ordering still survives for the third RDF [Fig. 5(c)] corresponding to Fig. 4(d). Finally, for the fourth RDF [Fig. 5(d)] corresponding to Fig. 4(e), peaks at long distances in the RDF disappear, but the peaks at short distances survive (i.e., moving Bragg glasses are transformed to moving vortex glasses). Therefore, shortrange ordering still exists in the second $2 \mathrm{D}$ flow state, which makes it quite different from the first 2D flow state, although their $c_{z}$ values and projections are similar. We perform a detailed measurement of in-layer ordering by calculating an $x y$-plane order parameter defined by

$$
c_{x y}=\int|1-g(r)| d r .
$$

As shown in Fig. 5, $c_{x y}$ is small for small driving. With increasing $f_{d}$, it increases sharply and reaches a peak at the same peak position in $c_{z}$. Then, comparing $c_{x y}$ with $c_{z}$, it decreases slowly, indicating that the in-layer ordering survives, which agrees with our previous finding from the analysis of the RDF.

With decreasing driving force $f_{d}$, one can still find a peak in $c_{z}$ and $c_{x y}$ [see Fig. 6(a)]. Comparing with the previous increasing $f_{d}$ case, the $c_{z}$ peak here is located at the same $f_{d}$ value but becomes much smaller. As shown in Fig. 6(b) at the peak, projections of pancake vortices form transverse stripes. Therefore, the formed vortex lines are deformed in the transverse direction.

To understand this unusual peak effect, we recall the previous studies about driving media in this so-called fast-sliding regime when $f_{d}>f_{p}$. On one hand, the random pinnings create thermal-like Langevin forces (TLFs) with an effective temperature $T_{c} \propto 1 / v^{2}$, which tends to melt the moving patterns [10]. On the other hand, they rotate the lattice orientation in the longitudinal direction [e.g., triangular lattices in Fig. 4(c)]. After the rotation, vortices move in static channels, i.e., an energy barrier locking transversal motion is created.

In Fig. 3(a), the saturated value of $c_{z}$ is smaller than 1 which results from the thermal-like fluctuations of vortex lines shaken by the TLF. For very strong pinning, the increased TLF is so strong that it is sufficient to tear the vortex lines, i.e., a dynamically induced melting of the vortex lines appears. Since the effective $T_{c}$ of the TLF decreases with increasing velocity $v \propto f_{d}, T_{c}$ takes the highest value when $f_{d}$ is just above $f_{p}$. In this case, 2D flow, instead of 3D flow appears.

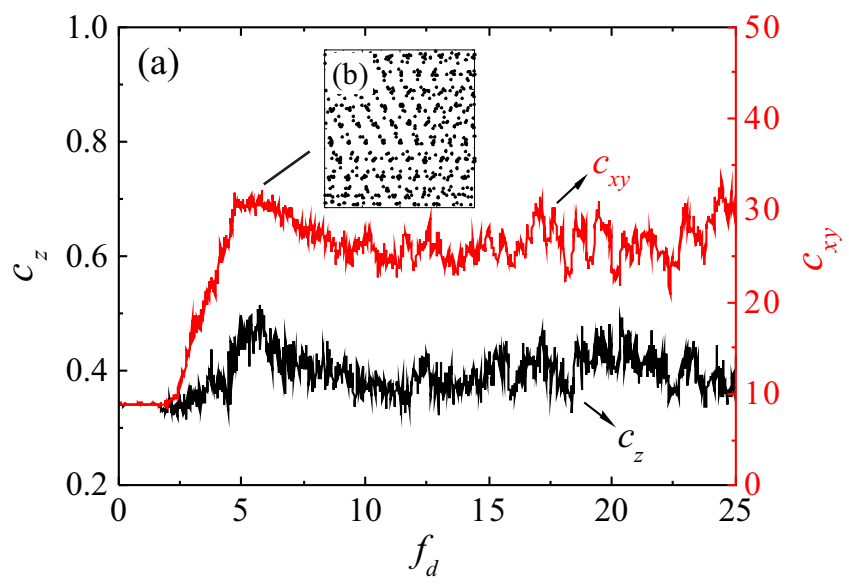

FIG. 6. (a) The correlation function $\left\langle c_{z}\right\rangle$ (black line) and $c_{x y}$ (red/gray line) versus driving force $f_{d}$ for coupling strength $s_{m}=4$ and pinning strength $f_{p}=2.7$. (b) Projections of pancake vortices' positions for $f_{d}=5.84$. 
Increasing $f_{d}$ at some critical point, the TLF becomes so weak that it leads to a $2 \mathrm{D}$ to $3 \mathrm{D}$ "crystallizing" transition. Note that the coupling of pancake vortices during the 2D-3D transition greatly reduces the effective pinning, which will also reduce the generated effective temperature $T_{c}$. This reduction is dominant as compared with that caused by an increase in $f_{d}$. Therefore, the $2 \mathrm{D}$ to $3 \mathrm{D}$ crystallizing transition is very sharp [see the sharp increase in $c_{z}$ in Fig. 4(a)], indicating that this transition is of first order. Further increasing $f_{d}$, the driving force, together with a dragging force created by random pinning, stretches the vortex lines in the longitudinal direction, which results in a stripelike projection shown in Fig. 4(d). Note that such a stretching effect also leads to the formation of moving stripes in driven systems with competing interaction [26]. The stretching of the vortex lines leads to a decrease in $c_{z}$. For even higher driving, these stretched lines are also melted. One possible reason is that the tilted stretched vortex lines have lower condensation energy than that of the straight lines because of weaker attraction due to longer intervortex distances after stretching. Therefore, the "melting temperature" $T_{m}$ resulting from their attractive interaction is also lower. The competition of the decreasing $T_{c}$ and reducing $T_{m}$ causes a $3 \mathrm{D}$ to $2 \mathrm{D}$ transition and leads to an interesting reentrant $2 \mathrm{D}$ flow phase. Note that only a slight decrease in $c_{z}$ is observed when the stretched vortex lines are melted, i.e., the transition is probably second order. Moreover, for the in-layer structure, it is determined by the competition of the TLF and in-layer repulsion. For those selected parameters, the TLF is much smaller than typical in-layer repulsive forces. Therefore, glass states are commonly found in the fast-sliding state. A peak in $c_{x y}$ appears at the same position as the peak in $c_{z}$, i.e., a strong correlation between in-layer ordering and interlayer ordering is revealed. The reason for this behavior is that, when optimal 3D states are formed, the TLF takes a minimal value because of the reduction of the effective pinning force. And the minimal TLF results in an optimal in-layer ordering, i.e., a peak in $c_{x y}$. For larger $f_{p}$, the TLF is so strong that the vortex lines are always melted. Therefore, only 2D flow can be found.

With decreasing $f_{d}$, the formation of transverse stripes is similar to that in Ref. [26]: comparing with the increasing $f_{d}$ case, the pancake vortices' transverse motion is locked since static channels are formed at higher driving. As a result, the required transverse motion of pancake vortices is obstructed, whereas the longitudinal motion is free. Thus, pancake vortices become closer to each other in the longitudinal direction but still remain apart in the transverse direction, which results in transversal stripelike projections as well as a smaller $c_{z}$ peak in Fig. 6.

\section{PHASE DIAGRAM}

The dynamical phase diagram on the plane of "interlayer coupling strength $s_{m}$ versus pinning strength $f_{p}$ " is shown in Fig. 7. For large $s_{m}$ and small $f_{p}$, the initial stable states are 3D pinned vortex lines. For very small $f_{p}$ with increasing $f_{d}$, these pinned vortex lines directly depin and form a 3D flow state (labeled 3DP-3DF). For stronger pinning, two additional intermediate dynamical regimes, 2D pinned and 2D flow, appear (labeled 3DP-2DP-2DF-3DF). The region where NDR appears is very small, and it is located inside

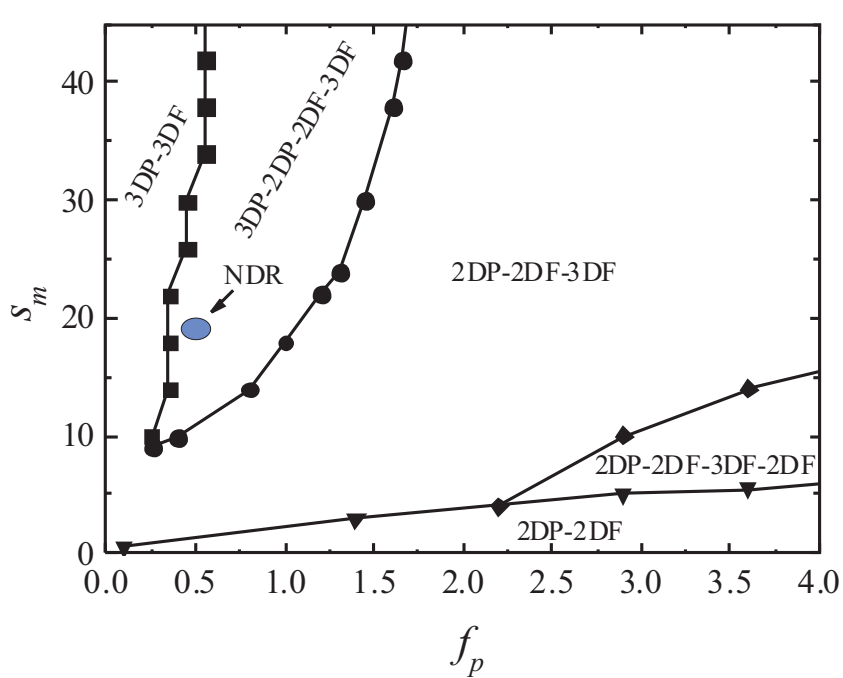

FIG. 7. The dynamical phase diagram in the plan of interlayer coupling strength $s_{m}$ versus pinning strength $f_{p}$ for increasing driving force $f_{d}$. 3DP, 2DP, 3DF, and 2DF are abbreviations for 3D pinned, 2D pinned, 3D flow, and 2D flow, respectively. The blue area marked by NDR is where we found NDR in which dynamical regimes are including 3D pinned, 3D flow, 2D pinned, and reentrant 3D flow.

the 3DP-2DP-2DF-3DF region. In this NDR region, a number of dynamical regimes are observed including: 3D pinned, 3D flow, 2D pinned, and another 3D flow (labeled 3DP-3DF2DP-3DF or simply NDR). For small $s_{m}$, the initial stable states are $2 \mathrm{D}$ pinned states. The corresponding dynamical regimes including: 2D pinned, 2D flow, and 3D flow (labeled 2DP-2DF-3DF) are commonly found for both weak and strong pinnings as well as for very strong pinning with large interlayer coupling strength. For very strong pinning and weak interlayer coupling strength, vortex lines in 3D flow are melted, i.e., only two dynamical regimes are found: $2 \mathrm{D}$ pinned and $2 \mathrm{D}$ flow (labeled 2DP-2DF). Finally, for very strong pinning and intermediate interlayer coupling strength, a peak effect in curve $c_{z}$ is revealed, indicating four dynamical regimes: 2D pinned, 2D flow, 3D flow, and reentrant 2D flow (labeled 2DP-2DF-3DF-2DP).

\section{CONCLUSIONS}

Using MD simulations, we analyzed the dynamics of driven pancake vortices in layered superconductors. Depending on the strength of the interlayer coupling, the static configurations are as follows: 3D pinned vortex lines or individually pinned pancake vortices. With increasing driving force, these 3D pinned vortex lines either directly depin for weak pinning, or they first transform to 2D pinned states, then to depinned 2D flow, and finally to 3D depinned flow for strong pinning. In the intermediate pinning region, a NDR effect is revealed associated with the following dynamical regimes: 3D pinned, 3D flow, 2D pinned, and another 3D flow. Note that here there is a reentrant pinned state as well as a reentrant $3 \mathrm{D}$ flow state. The $2 \mathrm{D}$ flow regime is missing here since the $2 \mathrm{D}$ pinned state at the transition point is a supercooled state, which can directly transform to a 3D flow state, jumping over the 2D flow state. 
For small interlayer coupling, generally, the 2D pinned state reorders to the $3 \mathrm{D}$ flow state via passing an intermediate 2D flow state. For extremely strong pinning, the thermallike Langevin forces generated by the random pinning in the fast-sliding regime lead to melting of the vortex lines, therefore, no 3D flow is formed. However, there exists an intermediate regime where this $2 \mathrm{D}$ flow first orders to $3 \mathrm{D}$ flow, and then vortex lines in this 3D flow state stretch in the longitudinal direction. These stretched vortex lines are then melted by the thermal-like Langevin force to uncoupled pancakes due to a decrease in the melting point. This discovered coupling-decoupling transition results in a peak effect in the $z$-direction order parameter $c_{z}$, which however cannot be observed experimentally by measuring $I-V$ curves. By calculating the in-layer RDF, we find the second (reentrant) 2D flow state, formed by melting of stretched vortex lines, which is partially ordered within the superconducting plane, whereas for the first 2D flow that appears just after depinning, it is completely disordered. With decreasing $f_{p}$, a small $c_{z}$ peak caused by the formation of vortex lines deformed in the transverse direction is revealed. This deformation is caused by the dynamical locking effect.
Based on these results, we summarized our main results in a dynamical phase diagram on the plane of interlayer coupling strength $s_{m}$ versus pinning strength $f_{p}$. Our findings can be useful for the analysis of vortex dynamics in layered superconductors. They can also be applicable for other layered systems, such as multilayered colloidal systems [41] or biological systems $[42,43]$. Similar phenomena may also appear in binary or multicomponent systems, such as vortices composed of different contributions in two-band or multiband superconductors where the dissociation of each component is similar to the decoupling transitions in layered systems.

\section{ACKNOWLEDGMENTS}

We acknowledge useful discussions with C. Olson Reichhardt. This work was supported by the Natural Science Foundation of Jiangsu Province (Grant No. BK20150595), National Natural Science Foundation of China (Grants No. NSFC-U1432135 and No. 11611140101). V.R.M. acknowledges support from the "Odysseus" program of the Flemish Government and Flemish Science Foundation (FWO-Vl), the FWO-Vl, and the Research Fund of the University of Antwerp.
[1] G. Piacente and F. M. Peeters, Phys. Rev. B 72, 205208 (2005).

[2] P. Glasson, V. Dotsenko, P. Fozooni, M. J. Lea, W. Bailey, G. Papageorgiou, S. E. Andresen, and A. Kristensen, Phys. Rev. Lett. 87, 176802 (2001).

[3] C. Reichhardt, C. J. Olson, R. T. Scalettar, and G. T. Zimányi, Phys. Rev. B 64, 144509 (2001).

[4] V. M. Vinokur and T. Nattermann, Phys. Rev. Lett. 79, 3471 (1997).

[5] S. Bhattacharya and M. J. Higgins, Phys. Rev. Lett. 70, 2617 (1993).

[6] U. Yaron, Nature (London) 376, 753 (1995).

[7] A. Duarte, E. Fernandez Righi, C. A. Bolle, F. de la Cruz, P. L. Gammel, C. S. Oglesby, E. Bucher, B. Batlogg, and D. J. Bishop, Phys. Rev. B 53, 11336 (1996).

[8] F. Pardo, F. De La Cruz, P. L. Gammel, C. S. Oglesby, E. Bucher, B. Batlogg, and D. J. Bishop, Phys. Rev. Lett. 78, 4633 (1997).

[9] F. Pardo, F. de la Cruz, P. L. Gammel, E. Bucher, and D. J. Bishop, Nature(London) 396, 348 (1998).

[10] A. E. Koshelev and V. M. Vinokur, Phys. Rev. Lett. 73, 3580 (1994).

[11] K. Moon, R. T. Scalettar, and G. T. Zimányi, Phys. Rev. Lett. 77, 2778 (1996).

[12] T. Giamarchi and P. Le Doussal, Phys. Rev. Lett. 76, 3408 (1996).

[13] L. Balents, M. C. Marchetti, and L. Radzihovsky, Phys. Rev. Lett. 78, 751 (1997).

[14] C. J. Olson, C. Reichhardt, and F. Nori, Phys. Rev. Lett. 81, 3757 (1998).

[15] B. Rosenstein and D. Li, Rev. Mod. Phys. 82, 109 (2010).

[16] C. Reichhardt, C. J. Olson, and F. Nori, Phys. Rev. Lett. 78, 2648 (1997).

[17] C. Reichhardt, C. J. Olson, and F. Nori, Phys. Rev. B 58, 6534 (1998).
[18] V. R. Misko, S. Savel'ev, A. L. Rakhmanov, and F. Nori, Phys. Rev. Lett. 96, 127004 (2006).

[19] V. R. Misko, S. Savel'ev, A. L. Rakhmanov, and F. Nori, Phys. Rev. B 75, 024509 (2007).

[20] E. H. Brandt and U. Essmann, Phys. Status Solidi B 144, 13 (1987).

[21] E. Brandt and M. Das, J. Supercond. Novel Magn. 24, 57 (2011).

[22] V. Moshchalkov, M. Menghini, T. Nishio, Q. H. Chen, A. V. Silhanek, V. H. Dao, L. F. Chibotaru, N. D. Zhigadlo, and J. Karpinski, Phys. Rev. Lett. 102, 117001 (2009).

[23] X. B. Xu, H. Fangohr, Z. H. Wang, M. Gu, S. L. Liu, D. Q. Shi, and S. X. Dou, Phys. Rev. B 84, 014515 (2011).

[24] H. J. Zhao, V. R. Misko, and F. M. Peeters, New J. Phys. 14, 063032 (2012).

[25] H. J. Zhao, V. R. Misko, and F. M. Peeters, Physica C 479, 130 (2012).

[26] H. J. Zhao, V. R. Misko, and F. M. Peeters, Phys. Rev. E 88, 022914 (2013).

[27] I. Giaever, Phys. Rev. Lett. 15, 825 (1965).

[28] R. Busch, G. Ries, H. Werthner, G. Kreiselmeyer, and G. Saemann-Ischenko, Phys. Rev. Lett. 69, 522 (1992).

[29] A. E. Koshelev, L. I. Glazman, and A. I. Larkin, Phys. Rev. B 53, 2786 (1996).

[30] C. J. Olson, G. T. Zimányi, A. B. Kolton, and N. GrønbechJensen, Phys. Rev. Lett. 85, 5416 (2000).

[31] B. Horovitz, Phys. Rev. B 72, 024519 (2005).

[32] M. Hilke, S. Reid, R. Gagnon, and Z. Altounian, Phys. Rev. Lett. 91, 127004 (2003).

[33] J. Lefebvre, M. Hilke, and Z. Altounian, Phys. Rev. Lett. 102, 257002 (2009).

[34] E. Babaev and M. Speight, Phys. Rev. B 72, 180502 (2005).

[35] S.-Z. Lin and L. N. Bulaevskii, Phys. Rev. Lett. 110, 087003 (2013). 
[36] C. J. Olson, C. Reichhardt, R. T. Scalettar, G. T. Zimányi, and N. Grønbech-Jensen, Phys. Rev. B 67, 184523 (2003).

[37] G. Blatter, M. V. Feigel'man, V. B. Geshkenbein, A. I. Larkin, and V. M. Vinokur, Rev. Mod. Phys. 66, 1125 (1994).

[38] J. R. Clem, Phys. Rev. B 43, 7837 (1991).

[39] N. Grønbech-Jensen, Comput. Phys. Commun. 119, 115 (1999).

[40] W. V. Pogosov, H. J. Zhao, V. R. Misko, and F. M. Peeters, Phys. Rev. B 81, 024513 (2010).
[41] E. C. Oğuz, A. Reinmüller, H. J. Schöpe, T. Palberg, R. Messina, and H. Löwen, J. Phys.: Condens. Matter 24, 464123 (2012).

[42] B. S. Gardiner, G. R. Joldes, K. K. L. Wong, C. W. Tan, and D. W. Smith, Comput. Methods Biomech. Biomed. Engin. 19, 1160 (2016).

[43] Nanobioelectronics Electrons, Biology, and Medicine, Nanostructure Science and Technology, edited by A. Offenhäusser and R. Rinaldi (Springer, New York, 2009). 